\title{
MobiDiagnosis: A Mobile Headache Characterization System
}

\author{
Dario Correal, Daniel Ramirez, Tatiana Hernandez, and Nicolas Diaz \\ Department of Systems and Computing Engineering \\ University of Los Andes \\ Cra 1E No 19A-40, Bogota, Colombia \\ Email:\{dcorreal, dc.ramirez147, t-hernan, n-diaz $\}$ uniandes.edu.co
}

\begin{abstract}
Certain diseases and clinical conditions can become serious public health issues. This is particularly true in neurology where chronic headache illness is a burden borne by a great extent of the population. Assertively diagnosing headaches requires the patient to fill out printed forms and collect daily information that may help identify all possible causes. Nevertheless, these printed forms can also become an obstacle to the patients' treatment. This article presents MobiDiagnosis, a mobile system intended to characterize headaches that allows for storing and on-line analysis of the symptoms related information.
\end{abstract}

Index Terms-Healthcare, Headache, Diagnosis, Mobility, Software Architecture.

\section{INTRODUCTION}

Certain diseases and clinical conditions become greater public health issues. In neurology, the prevalence ${ }^{1}$ of headache is far grater than in other contexts [1]. A Recent study[1] suggests that, $10 \%$ of the world population have been diagnosed with some kind of headache -according to this percentage, more than 700,000 people may be suffering from headaches only in the city of Bogota. The same studies estimate that almost $2.5 \%$ of these patients -close to 20,000 people in our environment- develop chronic pain each year and show little or no response to conventional treatments. Akin studies like the AMPP (American Migraine Prevalence and Prevention[2]) show prevalence of up to $11.7 \%$, and amongst those patients close to $53.7 \%$ display disabling symptoms. Headache disorders are among the most prevalent and costly diseases in the world[1].

The above indicates that headaches are a high prevalence illness among the world's population, and it causes loss of quality of life of half the people that suffer it. This makes the need for preventive strategies and therapies a must have for $25.7 \%$ of the chronic patients [2]. In most cases, the only means to manage and successfully treat headaches relies upon verifiable information that may enable a physician to characterize and keep track of the symptoms over time throughout the patients' evolution.

More often than not, headache patients are characterized using their clinical records along with diaries and printed forms where pain episodes are tracked down. This is where

\footnotetext{
${ }^{1}$ Ratio of ill people over the total population
}

the problem we aim to solve arises since it is quite common for patients not to have the printed forms handy when they need them the most, thus preventing their doctors from reading accurate and clear information that could be key to diagnosing or even preventing a further development of the illness. As if this were not enough, many patients are reluctant to fill out the printed forms and may fail to use them at crucial times.

This paper presents MobiDiagnosis, an ubiquitous application intended to characterize headaches, and designed so as to allow patients to keep track of the information their doctors crave for. MobiDiagnosis can be used on mobile devices such as cell phones, PDA's, and Internet Tablets. This application not only replaces printed forms, it also enables the patients to broadcast their personal information -which may be enriched with audio and video feeds-, or send out a distress signal with their current location so that they may be taken to a nearby ER.

MobiDiagnosis is currently being used at the Hospital Occidente de Kennedy Neurology service (Bogota, Colombia), where it has a test scenario of over 13,007 patients that begun external consulting during the year 2008. 3,960 of the 13,007 patients (close to $30.45 \%$ ) were forwarded to a specialist (see Figure 1). We believe a great deal of these patients had late diagnostics or were started on the wrong treatments for their condition, but we are unable to verify our belief since there aren't enough recorded symptoms to reach trustworthy statistical conclusions.

The remaining sections are as follows: section two describes the headache characterization requirements that were taken into account in our project. Section three depicts our solution's architecture. Section four gives account of our field experiences so far. Section five briefly discusses related work, and section six holds our conclusions and future work expectations.

\section{HEADACHE CHARACTERIZATION}

When dealing with headaches, a doctor's diagnose depends upon a great deal of variables but the most relevant are usually an accurate clinical history, careful physical exploration, paraclinical tests analysis and characterizing the evolution of symptoms. In the year 2008 the Hospital Occidente de 


\section{Prevalence of Diseases}

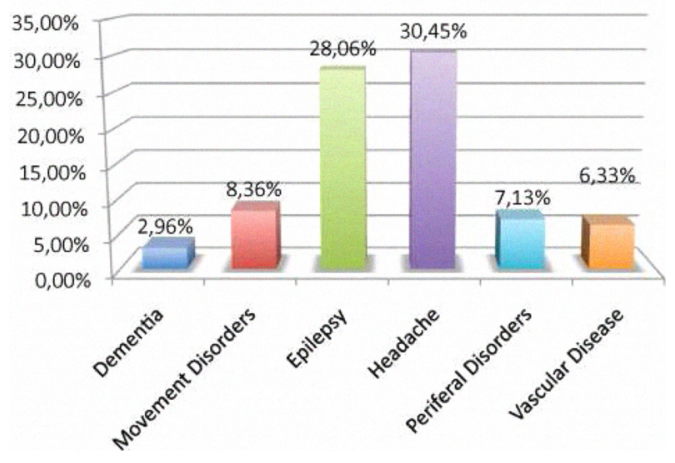

Figure 1. Diseases prevalance in a neurology service.

Kennedy's Neurology department treated 13,007 patients of which 3,960 (30.4\%) came to consult complaining about some kind of headache.

The information needed for accurate characterization comprises an episode's date and time, pain localization, pain intensity, use of medications, and response to medications. This data is crucial to assertively diagnose a patient since the finding or not of a specific symptom could foretell the primary or secondary causes of headache. Primary causes regard genetic or family features. Secondary causes are concerned with acquired diseases such as infections, vascular illness, or tumors. A patient's symptoms can place him in either one of the mentioned categories, but such symptoms may not arise when the patient consults a doctor and thus remain hidden until a certain catalyst (whether it be food, sleeping patterns, etc.) triggers them. Most traditional characterization models fail to provide a well-suited media where the patients can keep track of their symptoms.

These requirements are prone to change over time, given the fact that new symptoms may arise or that different kinds of analysis are better suited to address certain target populations. Nowadays, this kind of information is not available to doctors treating chronic headaches. All efforts spend on collecting this information should take into account the features of the target population.

\section{MobiDiagnosis ARCHITECTURE}

Given the great amount of information needed to identify all possible headache causes, proper diagnosis should require more information than just the symptoms or the patient's habits. Gathering not only the right amount of data, but also the level of detail present is of great value in order to achieve a successful headache diagnosis.

Whenever anyone is having a headache that person is likely to forget or not to notice all of the accompanying symptoms, which means that if they try to describe the situation afterwards then the information they provide is often inconsistent or incomplete. This is why we intend to use a mobile approach to the problem, so that the information may be gathered live whenever and wherever headaches happen to people. But even though the application will be always available, it remains crucial not to disregard the fact that the overall discomfort and pain caused by the headache may prevent patients from using the application while they are suffering an episode. Therefore, we need to allow the end user to create partial entries, and to edit and complement registered data with new entries. This is why an effort has been made in order to separate the gathered data and how it is presented to the user.

We are interested in analyzing the stored information, so that our mobile application may help a patient identify and avoid habits that may trigger or exacerbate headaches (i.e.: eating certain foods, engaging in strenuous physical activities, etc.). Gathered data will be stored at the mobile application where minor statistical analysis may be performed over information prone to such computations. We will trigger an analysis engine for every documented condition of interest and will use metadata to bind the analysis engine and the information it consumes. The analysis engine will be a simple business rule interpreter, and the target conditions it is able to diagnose are described as business rule sets. Several rule sets may be downloaded and installed from other application instances, or from a given URL, so that the analysis engine can be adapted to a patient's specific needs.

It is also desirable to be able to study headaches for epidemiological ends, but to do this we need more than one patient's data. All gathered information might be synced into the application server or other application instances, where it can be further analyzed. In spite of the existence of a central server, our architecture is not engraved in the client-server pattern. It is rather more a like the Peer-to-Peer (P2P) paradigm since the mobile end does not need the server side end in order to be fully functional.

\section{A. MobiDiagnosis: Mobile Application}

The patient side application simulates an agenda so as to provide a visually appealing and concise user interface. The remaining screens are intended to collect information about symptoms, medical treatment and medical prescriptions, as well as miscellaneous data such as eating habits, or accurate pain description (where does your head hurt? How bad would you say the pain is?) (see figure 2). We have also provided a voice recording facility, so that the patient may input data even when a headache makes it unthinkable to type text on the device. A voice registry can be marked as incomplete so that it can be reviewed later and linked to some other content node. All stored records can be synced to a mainframe warehouse where they can be further analyzed.

The application's components (see figure 3 ) are further explained in the following:

1) Content Management Component: Most of the data gathered by the application will be heterogeneous and will 


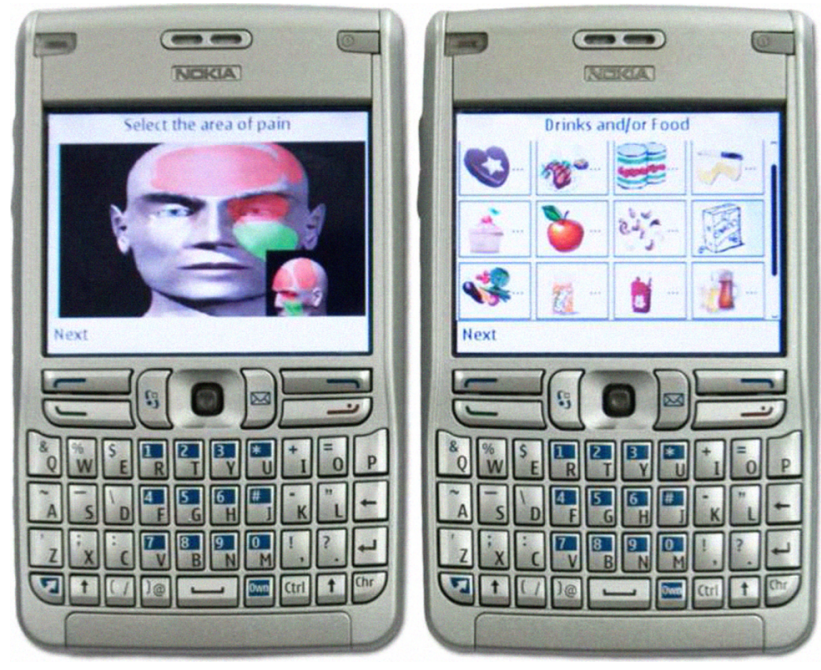

Figure 2. MobiDiagnosis cellphone application

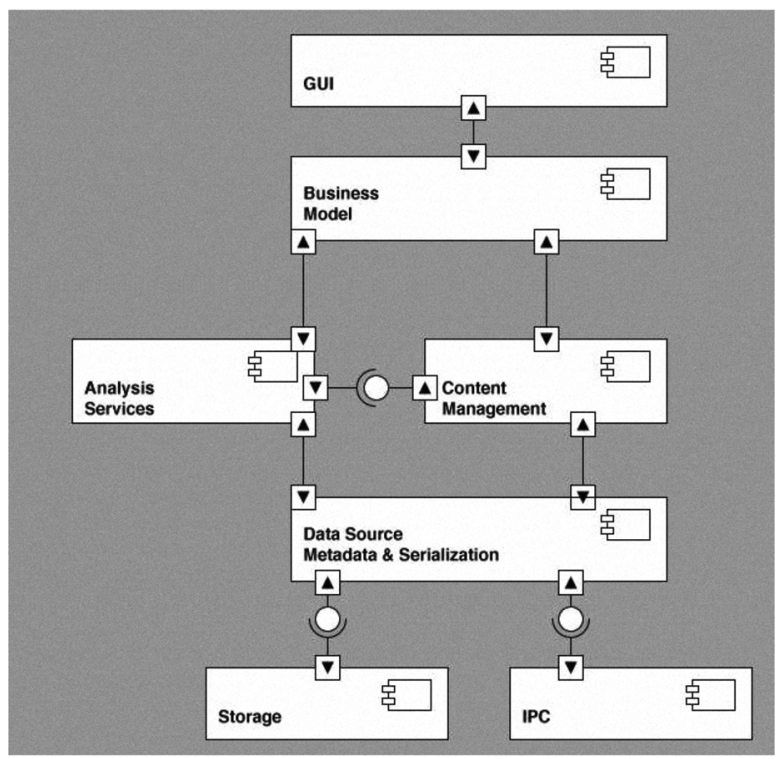

Figure 3. MobiDiagnosis Architecture: Components View

very likely change over time. Because we intend to collect live data we must take into consideration that people may be reluctant to use the application when they input data, thus the user interface needs to be friendly, intuitive, and allow for all records to be reviewed, edited and extended. Since these were amongst our main concerns, all information gathered from the user will be stored in a tree-like structure where each node will hold account of specific events and at a specific time. Each content node will be capable of storing either text, audio, image or video feeds and all content entries may be complemented by spawning child nodes where more information can be stored at. These content nodes are decoupled from the user interface, but the user interface is built out of components that specialize themselves in displaying a particular kind of content. Each node could be interpreted by a specialized component, a player. These players used by the user interface can take a node from the content tree and display it in whichever way best suits the addressed data whether it be audio, video, images or just text. The players can have implementation overrides that may help format the data they present to the user as "line view" when they are part of a list, "table cell view" when they are used to compose a table, or "full screen view" whenever a player has the whole screen for itself.

2) Data Source Component: Data analysis is crucial to our project because it is a feature that will encourage patients to keep tracking incidents and their daily habits, to hopefully improve their live style. The Data Source component is thus able to mark all content nodes using metadata so that the information within them may be sliced to provide an on demand analysis of headaches. All nodes holding headache pain intensity may be marked so that an on device stochastic analysis of headache intensity may be easily performed by implementing an analysis engine that traverses the content tree and consumes information -prone to statistical calculationsregarding pain intensity. For instance, whenever headache strikes, the content manager is able to identify business rules and store it in Analysis Services Component; therefore at a registration moment it can trigger alarms to notify the patient about possible pain catalysts regarding certain foods or strenuous physical activity. Suppose we define a business rule that casts cheese as a pain catalyst, and then the content manager will trigger a visual alarm if the patient records having eaten cheese.

Additionally, since large amounts of data will be stored on device as files representing the content nodes tree, to prevent these files from bloating the device, the Data Source component may choose to delete the files when they reach a certain age or right after the Inter-process Communication component synchronizes local records with the mainframe server.

3) Analysis Services component: In order to model and evaluate business rules, we have built the Analysis Services component (Figure 4). The business rules make our application flexible and can be used to suggest possible diagnostics based upon a patient's clinical records. As medical research evolves, it will be necessary to update or install different sets of business rules. To this end, all business rules can be installed, updated or removed by means of an HTTP request sent to the mainframe server or by local identification made by the Data Source Component.

4) Inter-process Communication component (IPC): This component comprises three major components: IO Streaming, Media Providers and Media Manager. The IO Stream component provides a custom stream implementation that holds the data to send, encapsulating it into a FIFO array queue. The Media Provider Component is in charge of managing communication channel definitions. It abstracts each channel protocol specific operation and exposes the common sending services with a generic interface. Finally, the Media Manager 


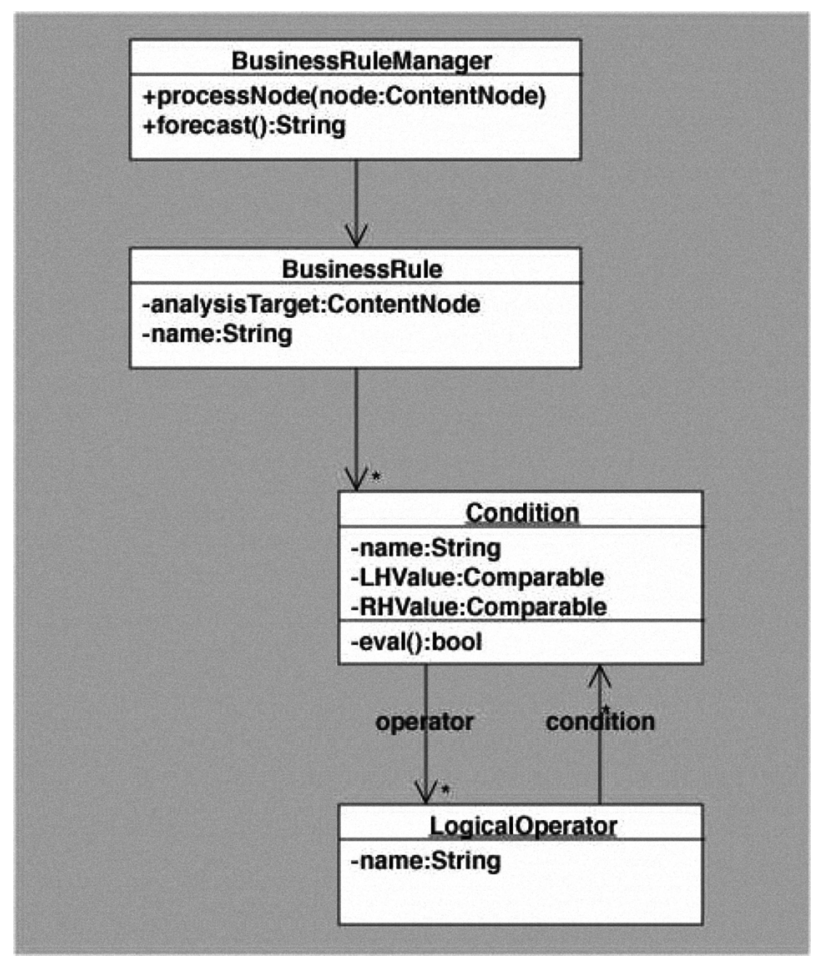

Figure 4. Analysis Services Component Structural View

is the component that makes the effective communication. It chooses the concrete communication provider, keeps endpoint definitions ${ }^{2}$, communicate with the endpoint and monitor each stream operation by the installs of probes ${ }^{3}$ over each IO Stream.

The Media Manager uses the Media Provider component to build a concrete media provider client and wrap them into I/O streams that are delivered to the application. Besides, a probe implementation can be overwritten; then, it can be configured to trigger a response to specific traffic conditions. For instance, if the data output rate falls below a certain threshold, a probe may decide to swap the streams' underlying media provider for a different implementation. Therefore, every time that a stream is shutted down, the Media Manager updates records of the media provider's performance by measuring the average of data throughput, and uses this information at stream startup to select an appropiate media provider with best historical performance.

5) P2P communication: Our application's capabilities can be further enhanced by providing a means of sharing stored information with other devices or a mainframe server where a greater amount of information can be consolidated and analyzed for demographic and forecast purposes. But even without the use of a mainframe server, several headache application instances could become slaves to a master instance, which is used by a doctor to check up upon his patients or

\footnotetext{
${ }^{2} \mathrm{~A}$ communication destination which offers services for a mobile device.

${ }^{3}$ Java classes that get the logic of tracking tasks. Those classes follow a general structure of service defined by a Java interface.
}

even ask a colleague for a second opinion or transfer a patient to another doctor.

Our application is mainly interested in sharing the content nodes it has stored for a patient, and to do this it simply endorses the fact that all data held within content nodes can be serialized as bytes. Thus to share a content node all that is needed is the availability of a byte stream where the data may be pushed into or pulled out of. There are many ways to build suitable byte streams in all JME devices, and HTTP is perhaps the most widely used since MIDP1. However the evergrowing device diversity makes it possible to use many other communication mechanisms like RFCOMM (radio frequency COM), IRDA, SMS, MMS and so forth. Using different communication mechanisms is very attractive because it will allow our application to take advantage of whatever resources are available to the device that hosts its execution, but it has one major downside: building a byte stream across any of these media may pose very different subtleties and in some cases (i.e.: SMS and MMS) the API does not work with byte streams. These limitations are addressed and screened by our communication's engine.

\section{B. MobiDiagnosis: Desktop Application}

The physician side of the application includes a website as a desktop version of the application (Figure 5), where all patient data may be reviewed and processed to display charts about headache treatment and the patient's response to the treatment. We address three main functional requirements: (R1) Search for a patient: Review a patient's clinical records using the patient's identification number. (R2) Review a patient's medical records for a given month: The physician can review a patient's medical records for a given time frame and use that data to build charts portraying pain intensity and pain relieve for the given medications; and (R3) Review a particular headache: Given a headache it is possible to review all related pain catalysts, symptoms and medications related to the episode.

1) Data management: The server has a 3-tier architecture: web, business and persistence tier. The last tier uses a flexible data structure to hold the nodes, which allows for enriching or even swapping symptom and pain trigger characterization. The data structure resembles the content node tree used by the device and is able to generate an ordered list of records for each patient.

Business Intelligence (BI) tools are used to process all stored information. This information allows a business expert to describe the symptoms and the effects of the treatments. BI tools are also used to update or install new business rules on the mobile devices by means of analyzing market basket, k-nearest neighbor, and cluster data, among others.

\section{Development strategy}

We developed the application in three phases: In phase 1, we asked target patients and their doctors for their interests 


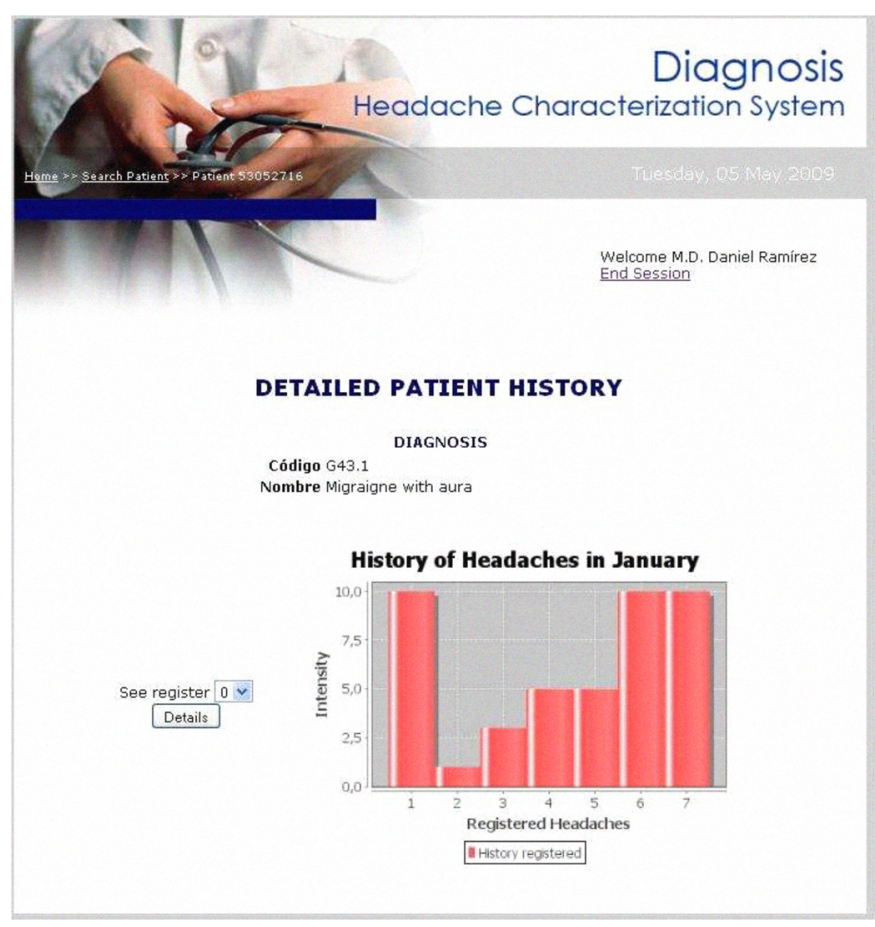

Figure 5. MobiDiagnosis Physician Desktop Application

and expectations about the application. During this phase we used polls and surveys to assess to what extent would our product find an embracing market. In phase 2, we developed the mobile application for the patients and begun gathering feedback and headache data. During this phase we performed usability tests for both mobile and website artifacts. Currently, we are in the middle of phase 3 , where the mobile application for the physician has yet to be completed. It will need P2P data exchange and a deeper and more wholesome analysis of all gathered information.

\section{EXPERIMENTATION}

We have implemented several tests to assess the usability and effectiveness of our framework in each of its development stages. The deployed applications are currently at the second stage, which includes collecting and characterizing gathered data. We used surveys to test the physicians' acceptance of the web tier with simulated input from 3 make-believe patients who suffered different symptoms and portrayed particular illnesses.

\section{A. Medical Web Application Usability Test}

1) Test Methodology: We used the Hallway Usability Test [3] methodology. This kind of tests are known to reveal up to $95 \%$ of an application's usability weak spots.

2) Test Development and Results: Five business experts (neurologists) filled out the surveys. All the questions in the survey were trying to measure usability under the following metrics: effectiveness, efficiency and satisfaction for each of the functional requirements tackled by the application.

We asked the doctors to answer questions regarding efficiency and effectiveness for each functional requirement. When they were done with this task we asked them to rate from 1 to 5 how satisfied they were with the implementation of the requirement and we also gathered their suggestions and improvement requests. The doctors' reactions while taking the tests were carefully observed by one of our staff members.

The general results are presented in the figure 6 . The navigation bar should be placed somewhere else, where it is more noticeable since only one of the doctors ended up using it. The doctors suggested to provide a quick launch menu for the navigation history, adding a headaches index meter and an information regarding whether or not the patient visited an ER.

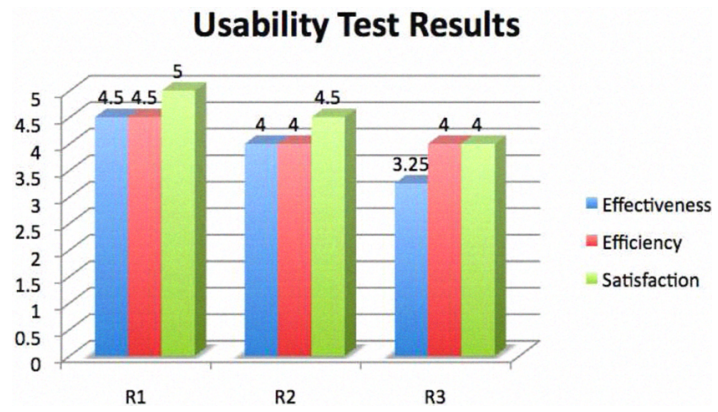

Figure 6. Usability Tests Results.

\section{B. Mobile Application Intention Survey}

1) Test Methodology: Likert's 5 level scales were used to achieve a test that could measure the acceptance and use proneness of the application among the study population[4]. The test was designed for an unbiased audience of men and women ages 21 to 40 who are currently under headache treatment.

2) Test Results: We interviewed 16 chronic headache patients and characterized their answers to our survey. It was clear that they had a strong tendency to resort to several medications, to visit ER's and also their longing for a tool that could help them track and manage their symptoms. 95\% of these patients own at least $1 \mathrm{JME}$ enabled device. You will find the result of the acceptance survey of mobile application at figure 7 .

The application was deployed on Nokia E62 cell phones with Wi-Fi Internet access. The staff member overseeing the patient's interaction with the device was satisfied with the results, but he also found plenty of room for improvement. For instance, the screen where the patients can identify the areas where they feel pain has slight performance issues and could be fine-tuned to avoid bloating the application. It would 


\section{MobiDiagnosis Use Intention}

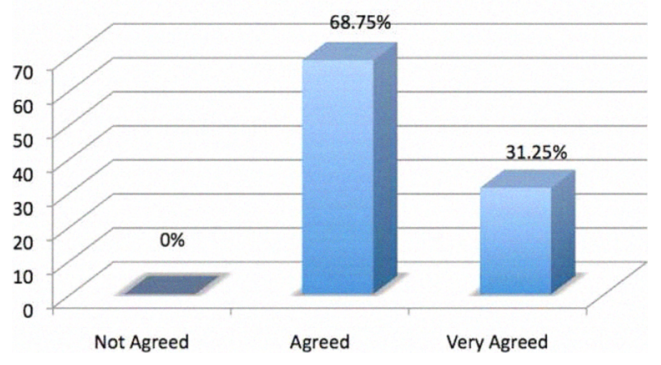

Figure 7. Intention Survey Results.

also be desirable to be able to use audio playback of previous recordings while fulfilling a form.

\section{RELATED WORK}

You may find an alternate approximation to symptom characterization on the article written by T. Barbosa [5], in which a Bayes net is woven using a symptom tree. The symptom tree represents a particular diagnosis, where each node stands for a question that determines (or rules out) an element that is to be characterized. This method has one major setback: keeping the information in the Bayes net up-to-date is a shore that can only be appointed to a business expert.

In [6], the authors present the Shimane Community Network System (Shimane CNS), a tool for healthcare communication between Shimane Medical University Hospital and home doctors. Based on this tool, the authors developed RHINOS, an e-decision support tool which diagnoses clinical cases on headache or facial pain. The main difference between MobiDiagnosis and Shimane CNS is that in Shimane, mobile devices are used only as terminals to interact with a main server via HTTP. In our work, mobile devices play a different role in the architecture, they are used as computational and independent units capable of make decisions based on business rules.

MobiDiagnosis has a clear advantage over previous works on headache characterization: it offers data mining tools. MobiDiagnosis is also more compelling to the end user (the patient) because it does far more than just store and share information; it is able to provide live feedback based upon the evaluation of flexible and ever evolving business rules. MobiDiagnosis has a friendly and intuitive user interface, which may make it easier for the patients to take a more proactive stance in their own treatment.

\section{CONCLUSIONS AND FURTHER WORK}

Our work is mainly concerned with the design and development of a mobile platform to enable for accurate symptom characterization when dealing with chronic headache patients. We have proposed an architecture that can manage flexible and ever-changing information schemes, and consolidate data across several platforms and communication channels. All gathered information could be used for business domain analysis.

So far, we have partially validated the extent to which our work has touched the medical community (both doctors and their patients) by means of the tests described at section 4 . Nevertheless, we cannot disregard the fact that most of the patients have happily embraced the mobile application and the medical team remains optimistic about the features offered to them.

After fulfilling a test plan for the mobile application, we still lack feedback over a wider range of devices before extending the application's services to a greater number of patients. It is also desirable to test our pilot for a longer time span so that we may conclusively assert and measure MobiDiagnosis' impact on headache treatment.

We also want to stress the fact that we are enabling people to collect information using non-conventional media (i.e.: audio and video feeds) that are easier to use in mobile platforms. This and the ability to share live information with a physician bring new horizons related to medical treatments that are yet to be explored.

Our future work will be dedicated to finishing the third phase of the project, and implementing registry sharing between the doctor and the patient (master and slave application instances).

\section{ACKNOWLEDGMENT}

The authors wants to thank Dr. Fidel Sobrino, Dr. Javier Vicini, Dr. Carlos Niebbles and Dr. Ramon Quintero who supported the project by providing medical context advice, patients' insight and careful statistics. This work was also possible thanks to the Hospital Occidente de Kennedy and their constant counseling.

\section{REFERENCES}

[1] L. S. Rigmor Jensen, "Epidemiology and comorbidity of headache," The Lancet Neurology, vol. 7, no. 4, pp. 354-361, April 2008.

[2] R. Lipton, M. Bigal, M. Diamond, F. Freitag, M. Reed, and W. Stewart, "Migraine prevalence, disease burden, and the need for preventive therapy," Neurology, vol. 68, pp. 343-349, 2007.

[3] J. Nielsen, Usability Engineering (Interactive Technologies). Academic Press, 1993.

[4] S. R. Sundaresan Ram, "Validation of expert systems for innovation management: Issues, methodology, and empirical assessment," Journal of Product Innovation Management, vol. 13, pp. 53-68, 2003.

[5] A. Jose, T. de A Barbosa, I. Sene, A. da Rocha, L. da S Castro, F. de O Nascimento, J. Carvalho, and $\mathrm{H}$. Carvalho, "A framework for automated evidence gathering with mobile systems using bayesian networks," Proceedings of the 29th Annual International Conference of the IEEE EMBS, 2007. [Online]. Available: http://ieeexplore.iee.org/ stamp/stamp.jsp?tp $=\&$ arnumber $=4353130$

[6] S. Tsumoto, S. Hirano, H. Abe, H. Nakakuni, and E. Hanada, "Clinical decision support based on mobile telecommunication systems," in WI '05: Proceedings of the 2005 IEEE/WIC/ACM International Conference on Web Intelligence. Washington, DC, USA: IEEE Computer Society, 2005, pp. 700-703. 